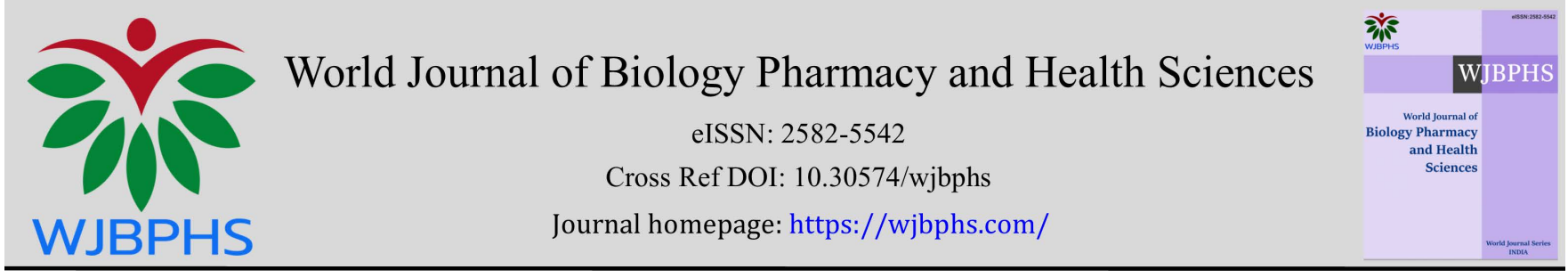

(RESEARCH ARTiCLE)

\title{
Restoration of the anterior guide with the fixed prosthesis
}

\author{
Sihem Hajjaji *, Shayma Karray, Hayet Hajjemi and Abdelatif Boughzela \\ Department of dentistry, Farhat Hached university hospital, sousse/ Tunisia.
}

World Journal of Biology Pharmacy and Health Sciences, 2021, 08(02), 019-025

Publication history: Received on 10 October 2021; revised on 17 November 2021; accepted on 19 November 2021

Article DOI: https://doi.org/10.30574/wjbphs.2021.8.2.0122

\begin{abstract}
The anterior guide is a functional entity, which is part of the set of guidance provided by the dento-dental joints to the mandibular kinematics, essential for the establishment of harmonious occlusal relations.

The anterior guide is a key to understanding and reconstructing the occlusion, playing both a role of protecting the posterior teeth and guiding the functional movements of the mandible. Currently, it also represents an "exteroceptive feeler" creating a cone of access to the occlusion in intercuspation. The restoration of functional and protective anterior guidance is therefore essential for the balance of the masticatory system. This rehabilitation can only be achieved by examining and accurately diagnosing the occlusion relationships maintained by the anterior teeth, associated with a reasoned therapeutic approach validated by a test phase. After a brief description of the anterior function and its role, this article aims to detail, through a clinical case, the procedure to follow for the restoration of a functional anterior guide.
\end{abstract}

Keywords: Anterior guide; Occlusion; Bite curve; Incisor slope; Prosthesis

\section{Introduction}

The health of the manducatory system depends on the balance and functional harmony of its various dental, periodontal, neuromuscular and joint components. The anterior teeth have a privileged role in the balance of the masticatory system [15]. Indeed, they participate in the static mandibular wedging function, the dynamic function of nutrition and phonation and the kinematic function of guiding the mandible [1]. They protect dental, periodontal, muscle and joint structures by supporting mandibular movements. This care is done thanks to the particular anatomy of this group of teeth, but also and above all thanks to a proprioceptive component, responsible for the phenomena of adaptation and regulation of masticatory cycles. The previous guide then takes on all its importance [5]. This work aims to highlight the interest of a functional anterior guide and the means to achieve it in the context of a prosthetic restoration by fixed prosthesis [21].

\section{What is the anterior guide?}

The notion of anterior guide is based on the gnathological concept of mutual protection, i.e. the posterior teeth should be protected from transverse (horizontal) forces during lateral movements of the mandible [23]. The premise being that posterior teeth only tolerate axial (vertical) forces well and, conversely, shear forces are liable to cause all kinds of damage (wear, cracks, fractures, periodontal damage, etc.). The anterior guide corresponds to a summation of guiding slopes which direct the mandibular movements eccentric by the six antagonistic anterior teeth [2,9]. It begins at the point of IOM (maximum intercuspid occlusion), and allows mandibular movements (propulsion, right and left laterality). The idea was supported by the fact that when the mandible makes lateral or anterior movement from the

\footnotetext{
${ }^{*}$ Corresponding author: Sihem Hajjaji

Department of dentistry, Farhat Hached university hospital, sousse/ Tunisia.

Copyright (C) 2021 Author(s) retain the copyright of this article. This article is published under the terms of the Creative Commons Attribution Liscense 4.0.
} 
centered occlusion position, there is a "protective" disocclusion for the posterior teeth [22]. For gnathologists, the posterior determinants of occlusion (TMJ) must be in perfect harmony with the anterior determinants of occlusion (tripodism, cusp angles, canine and incisor slopes). [6]

\section{Evaluation of the anterior guide}

It is necessary to assess the functional value of the existing anterior guidance compared to an ideal academic reference model. The occlusal architectural elements of the ideal anterior guide are both diagnostic criteria and rules for occlusal construction [11].

Functionally, the examination of the anterior guide involves examining the occlusal contacts. Contacts in OIM should be symmetrically distributed about the mid-sagittal plane, of lower intensity than at the posterior level [25].

In propulsion, the free edge of the mandibular incisors slides from the contacts of the IOM in continuous linear paths, or relays, symmetrical along the maxillary guiding surfaces, to the incisal (or incisor-canine) end to end [7]. This slippage should cause the cuspid teeth to disengage. (Fig. 1)

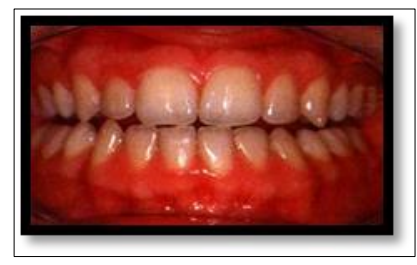

Figure 1 Propulsion movement

Laterally, the mandibular canine points slide on the mesial pan of the maxillary canine. This is the canine function where inter-canine contact is maintained from the start of the movement of the mandible to the canine end-to-end, with immediate disengagement of the working and non-working sides (fig. 2). In the event of group function, several teeth (posterior and / or anterior) adjacent to the canine are in contact over part or the entire movement path, and help guide the mandible (fig. 3). [7, 13]

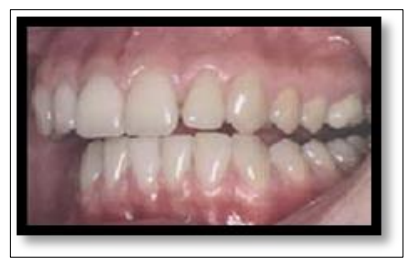

Figure 2 Canine protection

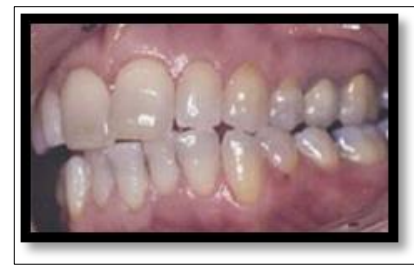

Figure 3 Group protection

The evaluation of the previous guide thus makes it possible to determine whether the reports of the existing previous guide should be kept or not. The guide can be considered functional and should be kept if it has the main predetermined characteristics. Otherwise, it must be recreated [4].

Thus, the guide can be considered as functional (fig. 4) when it does not exist (gap for example) [3]

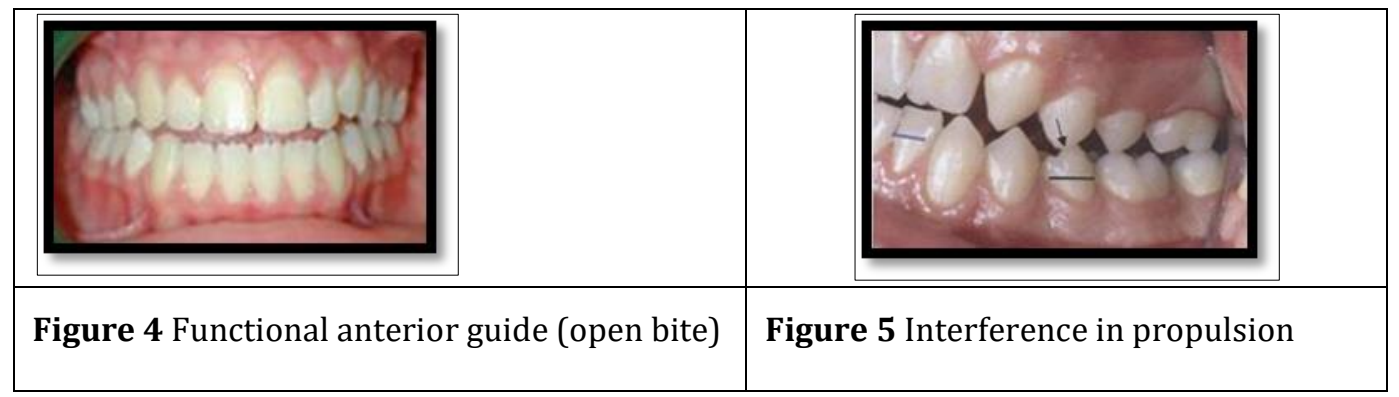


The guide is said to be dysfunctional (fig.5) when it exists but it constitutes an obstacle to mandibular movements (absence of immediate disocclusion during propulsive movement and lateralization in the presence of interference). [10]

\subsection{Rehabilitation of the anterior guide : clinical case}

The rehabilitation steps of the anterior guide will be detailed through a clinical case. A step-by-step approach to the case ensures a logical and systematic approach.

Presentation of the case and evaluation: (fig. 6a, b, c, d)

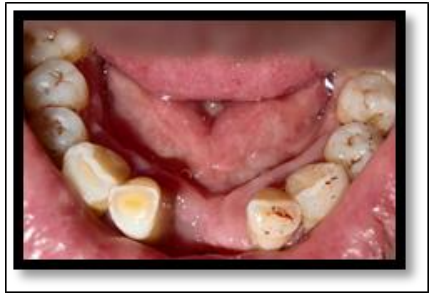

Figure 6a Initial situation in the mandible

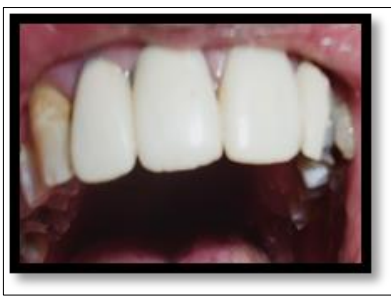

Figure 6 b, c Initial situation in the jaw
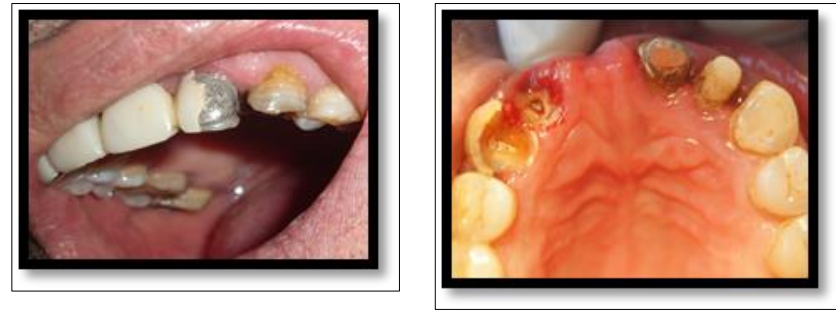

Figure 6 d Situation after removal of the maxillary bridge

This is a 56-year-old patient who consults for the replacement of 41 and 31 absent due to trauma.

The clinical examination shows the absence of 41 and 31, a bridge replacing the 21 having as supporting teeth the 11, 12 and 22, extensive caries on the 23 and 22. The radiological examination shows a bone lysis which reaches the 1 / 3 apical of 42. Examination of the occlusion reveals a dysfunctional anterior guide.

Our therapeutic project will therefore aim to restore functional anterior guidance allowing freedom of mandibular movements, without anterior or posterior interference, while maintaining periodontal health.

Our therapeutic project will therefore aim to restore functional anterior guidance allowing freedom of mandibular movements, without anterior or posterior interference, while maintaining periodontal health.

\subsection{Pre-prosthetic treatments and sanitation : (fig. 7.a, b)}

Root planing scaling was necessary to cleanse the oral cavity and prepare the site for the future prosthesis. The extraction of 42 followed significant bone lysis (1/3 apical), as well as increased mobility (degree 2). The 22 and 23 benefited from coronary caries treatment, then endodontic treatments, to end with a coronary elongation, the objective of which is to restore the biological space and provide sufficient coronary walls to maintain the future prosthesis.

Finally, the coronal-radicular reconstitution of 11,22 and 23 by metallic inlaycores completes this first phase of treatment.

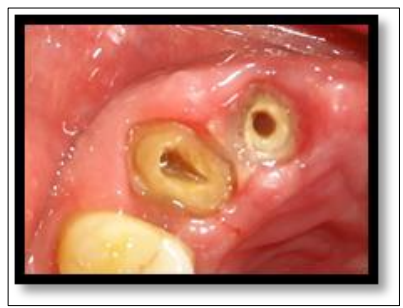

Figure 7a Coronary elongation $(22,23)$

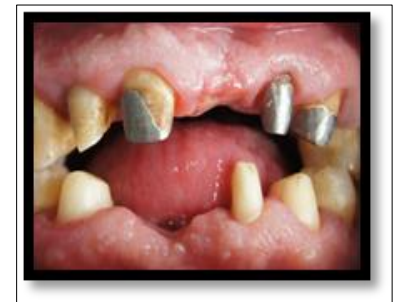

Figure 7b Reconstruction of 11, 22 and 23 


\subsection{Elaboration of the prosthetic project (wax up) : (fig. 8.a, b)}

It corresponds to the wax materialization of the prosthetic project. Diagnostic waxes (wax-up) and mounting on an articulator are carried out according to the ideal occlusion plane. The lingual surfaces of the teeth will be fitted with wax taking into account all the clinical data $[18,24]$.

Establishing a suitable occlusion (OIM and anterior guidance) on the diagnostic wax-up is of great importance as the latter will be used to fabricate a provisional restoration that reproduces the same occlusal and aesthetic characteristics.
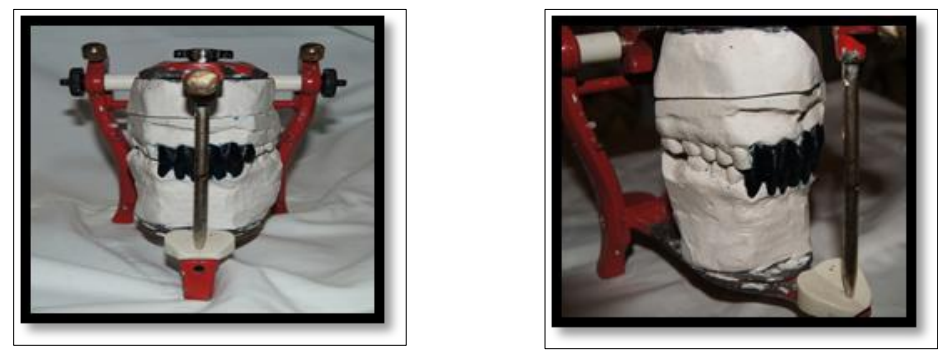

Figure 8 a, b Elaboration of the prosthetic project

\subsection{Making provisional prostheses: (fig. 9.a, b, c, d)}

It corresponds to the transfer to the mouth of the modifications obtained on study models. These temporary prostheses are made from diagnostic waxes [17].

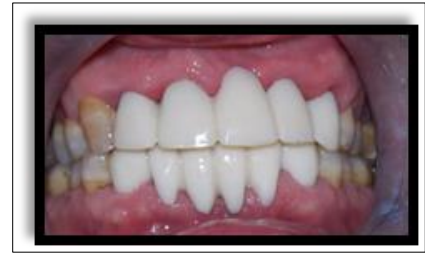

Figure 9.a Provisional prosthesis in static occlusion

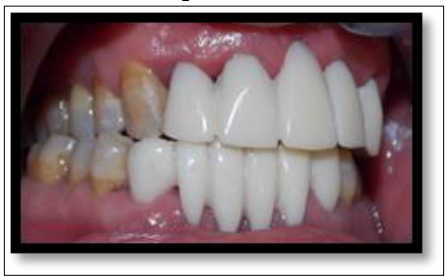

Figure 9.c Right laterality

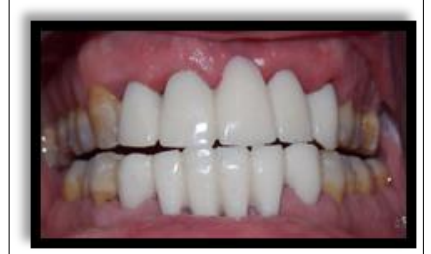

Figure 9.b Provisional propulsion prosthesis

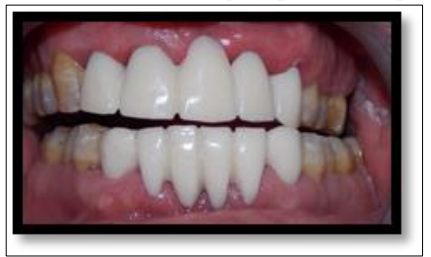

Figure 9.d Left laterality

The palatal surfaces of the provisional crowns are then modeled by addition or grinding of resin in order to best approximate, the morphology adapted to a correct anterior guide.

\subsection{Registration of the anterior guide (animation of the incisor table)}

When the anterior guidance is perfectly restored in the mouth using the provisional prostheses, we then record it in order to make our final prostheses:

- After validation of the provisional prostheses in the mouth, maxillary and mandibular impressions are taken with the two provisional bridges in the mouth (fig. 10.a, b). The casting of these impressions allows us to obtain two models, which are then mounted on an articulator in the maximum intercuspidation position (fg.11).

- Subsequently, we will place the resin on the incisor table, close the upper branch of the articulator, which will therefore pass through this resin, and we will immediately start guiding the teeth in propulsion and laterality until the resin sets [14]. 
- The rod leaves traces that form an envelope of functional movements : this is the Gysi arc (fig. 12.a, b, c, d). It is this functional incisor table made from temporary prostheses that is used to develop the final prosthesis.

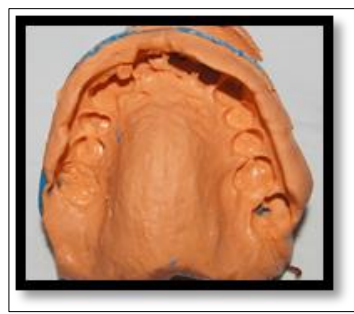

Figure 10. a, b Maxillary and mandibular impressions with the provisional prostheses in place

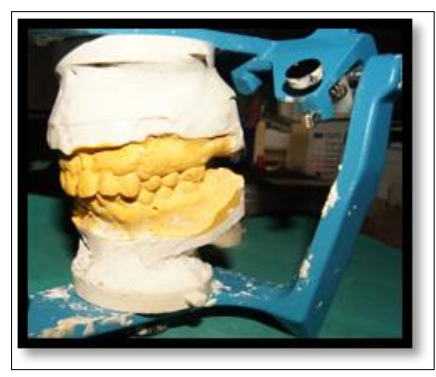

Figure 11 Assembly of models on articulator

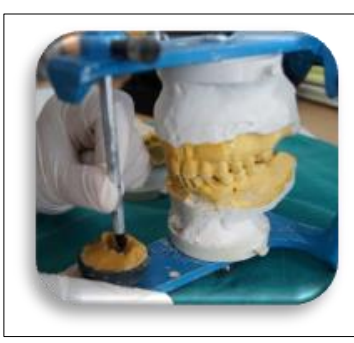

Figure 12.a Propulsion guidance

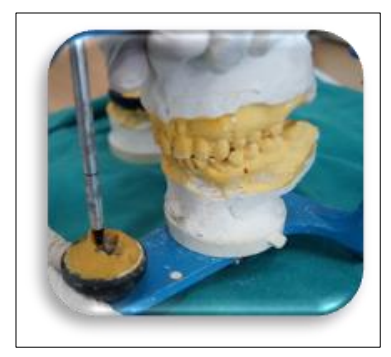

12.b Right laterality

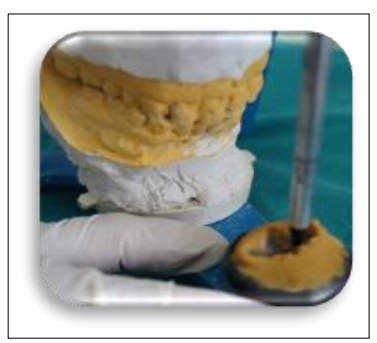

12.c Left laterality

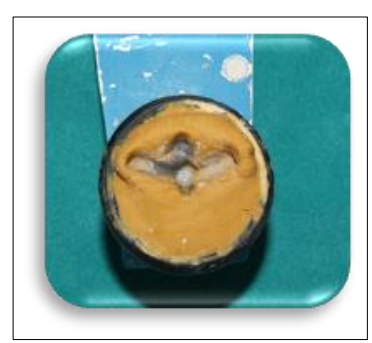

12.d GYSI arc

\subsection{Making the final prostheses}

After recording the anterior guide, the practitioner takes impressions of the preparations in the mouth (fig. 13), their casting (fig. 14) and their mounting on the articulator. Subsequently, it is up to the prosthetist to carry out the final prostheses, of course taking into account the previously recorded and validated incisor table (fig. 15).

\begin{tabular}{|l|l|l|}
\hline Figure 13 Imprint of preparations & Figure 14 Casting of the impression & \begin{tabular}{|c|c|}
\hline Faxes taking into account the incisal \\
table
\end{tabular} \\
\hline
\end{tabular}

\section{Conclusion}

The restoration of a functional and protective anterior guide is an essential condition for the success and sustainability of any prosthetic treatment [19].

This objective can only be achieved by examining and accurately diagnosing the occlusion relationships maintained by the anterior teeth, associated with a reasoned therapeutic approach. 
Programming the incisor table according to individual data ensures the precise transfer of guidance parameters to the laboratory for optimal prosthetic integration $[13,20]$.

However, it remains a very delicate work, insofar as it is necessary to create a perfect harmony between sometimes contradictory purposes (aesthetic and functional). [12]

\section{Compliance with ethical standards}

\section{Disclosure of conflict of interest}

No conflict of interest

\section{Statement of informed consent}

There is no research that has been done. The clinical case was treated according to the rules of the department

\section{References}

[1] Nelson SJ. Wheeler's dental anatomy, physiology and occlusion. Philadelphia: Saunders. 2014.

[2] Casteyde JP. The occlusion of the canine. Importance, adjustement options, risks and precautions, Actualités Odonto-Stomatologiques. 2008; 244: 352-366.

[3] 3. Zachrisson BU. Esthetic factors involved in anterior tooth displayand the smile ; vertical dimension. J Clin Orthod 1998; 32: 432-44.

[4] Ré JP, Perez C, Giraudeau A, Ager P, El Zoghby AE, Orthlieb JD. Reconstuction of the curve of Spee. J stomat. 2008; 105: 29-32.

[5] Paterson AH. Influences of mandibular movements on balanced occlusion. The Journal of American Dental Association. 1928; 15: 1118-23.

[6] Lahori M, Siddhart h, Chakraborty DS. Virtual articulators: changing trends in prosthodontics. Guident. 2013; 6: 10-28.

[7] Gray H. Anatomy of the human body. New york : édition Léa and Febiger. 1918.

[8] Paterson AH. Construction of artificial dentures. The dental cosmos. 1923; 65: 679-89.

[9] Ruge S, Kordass B. Initial results from computerized visualisation of dynamic occlusion.Int J Comput Dent. 2008; 11: 9-19.

[10] Singh N, Dandekeri S, Shenoy K, Bhat V. Digital articulators: a promising technology of the future . International journal of dental and medical research. 2014; 1: 98-102.

[11] Ré JP, Giraudeau A, Orthlieb JD, Zoghby AE. Inclination of mandibular canine in relation to the condylo-occlusal line. J. stomat. Occ. Med. 2008; 1: 16-20.

[12] Fukoe H, Basili C, Slavicek R, Sato S, Akimoto S. Three dimensional analysis of the mandible and the occlusal architectureof mandibular dentition. J Stomat. Occ .Med. 2012; 5: 119-129.

[13] Mchorris WH. Occlusion with particular emphasis on the functionnal and parafunctionnal rôle of anterior teeth. J Clin Orthod. 1979; 13: 606-620.

[14] Stuart CE. Good occlusion for natural teeth. J prosthet dent. 1964; 14: 716-724.

[15] Page H. The occlusal curve. dental digest. 1952; 1: 19-21.

[16] Iraqui 0, Berrada S, merzouk N, Abdedin A. Anterior guide, physiological requirements and prosthetic restauration. Actualités Odonto-Stomatologiques. 2009; 245: 83-98.

[17] D'Amico A. «Functionnal occlusion of the natural teeth of man ». The journal of prosthetic dentistry. 1961; 11(5): 899-915.

[18] Pasricha N, V Sidana, S Bhasin, M Makkar. «Canine protected occlusion ». Indian journal of oral sciences. 2012; 3(1): 13. 
[19] Dawson PK. Solving occlusal problems through programmed treatment planing. In: Evaluation, diagnosis and treatment of occlusal problems. St louis: Mosby. 1970; 387.

[20] Dos Santos, J Jr. Occlusion, principles and treatment. 2007; 232.

[21] Priyanka Mall BM. Rehabilitation of antérior teeth with customised incisal guide table.. 2013.

[22] Hans Muller C. Registration of occlusion by buccal scan in Cerec software version 3.80. International journal of computerized dentistry. 2010; 13(3): 265-273.

[23] Kerstein R. Computer occlusal analysis technology and Cerec case finishing. International journal of computerized dentistry. 2008; 11(1): 51-63.

[24] Next generation Cerec » International journal of computerized dentistry. 2009; 12(1).

[25] Papazian S, Morgano S. A procedure for making an interocclusal record without the use of record bases for a combined fixed/removable prosthesis. The journal of prosthetic dentistry. 1998; 79(2): 220221. 